\title{
The stats are in: an update on statin use in COPD
}

\author{
This article was published in the following Dove Press journal: \\ International Journal of COPD \\ 22 October 2015 \\ Number of times this article has been viewed
}

\author{
Alexa A Carlson' \\ Ethan A Smith ${ }^{2}$ \\ Debra J Reid' \\ 'Department of Pharmacy and Health \\ System Sciences, School of Pharmacy, \\ Northeastern University, Boston, MA, \\ USA; ${ }^{2}$ Department of Pharmacy, Beth \\ Israel Deaconess Medical Center, \\ Boston, MA, USA
}

\begin{abstract}
COPD is a chronic inflammatory disease of the lungs associated with an abnormal inflammatory response to noxious particles, the most prevalent of which is cigarette smoke. Studies have demonstrated that cigarette smoking is associated with activation of the bone marrow, and chronic smoking can lead to the inflammatory changes seen in COPD. Due to the inflammatory nature of the disease, medications affecting the inflammatory pathway may have clinical benefit and are being evaluated. One such class of medications, HMG-CoA reductase inhibitors, have been evaluated in the COPD population. Early studies have suggested that HMG-CoA reductase inhibitors have a variety of benefits in COPD including improvements in inflammatory markers, exacerbation rates, and mortality rates. However, the majority of this data comes from retrospective cohort studies, suggesting the need for randomized controlled trials. Recently, two randomized controlled trials, STATCOPE and RODEO, evaluated the benefit of HMG-CoA reductase inhibitors in the COPD population and found no benefit in exacerbation rates and vascular or pulmonary function, respectively. These results are reflected in practice guidelines, which do not support the use of HMG-CoA reductase inhibitors for the purpose of reducing COPD exacerbations.
\end{abstract}

Keywords: chronic obstructive pulmonary disease, statins, HMG-CoA reductase inhibitors

\section{Introduction}

COPD is a chronic respiratory condition associated with enhanced inflammatory response of the airways to noxious particles, ${ }^{1}$ the most prominent of which is cigarette smoke. Cigarette smoking has been associated with contribution to the inflammatory response in the lungs and resultant stimulation of the bone marrow. A study by van Eeden and $\mathrm{Hogg}^{2}$ sought to assess the effects of smoking on the bone marrow amongst 38 adult smokers and 15 matched controls. Compared to the control group, the smoking group had a higher mean total leukocyte $(P=0.001)$, polymorphonuclear leukocyte $(P=0.003)$, and band $(P=0.01)$ count. This increase in polymorphonuclear leukocytes and bands, along with elevated levels of 1-selectin, an adhesion molecule, and MPO, an enzyme found in the circulating polymorphonuclear cells in the smoking population compared to the control population $(P<0.05$ for both), demonstrates bone marrow stimulation in response to chronic smoking. ${ }^{2}$ Similar results demonstrating inflammation have been reported elsewhere. ${ }^{3}$ Persistent use of cigarettes over time can lead to a chronic inflammatory response in COPD. ${ }^{4}$ This inflammatory response leads to the long-term physiologic changes seen in COPD. ${ }^{1,5-11}$ By measuring CRP and IL-6 values of 983 subjects in Iceland, Thorleifsson et al found both values to be independently related to lower forced vital capacity and forced expiratory volume in 1 second values. Both inflammatory markers were also found to be associated with smoking pack-years, further supporting that chronic smoking and COPD result
Correspondence: Alexa A Carlson Department of Pharmacy and Health System Sciences, School of Pharmacy, Northeastern University, 360

Huntington Avenue, R218TF, Boston, MA 02II5, USA

Tel + I 6173733520

Fax +I 6173737655

Email a.carlson@neu.edu 
from inflammatory processes. ${ }^{12}$ This association of elevated inflammatory mediators in COPD has been found in a number of other studies. ${ }^{13-16}$ Systemic inflammation becomes important as elevated plasma CRP has been associated with increased cardiovascular risk ${ }^{17}$ thereby supporting systemic inflammation as a potential focus of treatment.

\section{Anti-inflammatory effects of HMG- CoA reductase inhibitors}

Due to the inflammatory nature of COPD, medications targeting the inflammatory process should have a theoretical benefit. One such class of medication, the HMG-CoA reductase inhibitors, or statins, are primarily utilized for the management of lipid and cardiovascular disorders. Statins have also been shown to have pleiotropic and anti-inflammatory properties which have demonstrated benefits in a number of disease states including but not limited to those of infectious, ${ }^{18}$ cardiovascular, ${ }^{17,19-23}$ and pulmonary origin. ${ }^{24}$ Some proposed mechanisms behind this effect include interference with cellular adhesion ${ }^{25}$ and proliferation, ${ }^{24}$ increased apoptosis, ${ }^{26}$ and alteration of cytokine release. ${ }^{27}$ Multiple studies in cardiovascular patients have demonstrated the ability of statins in reducing inflammatory mediators like CRP, independent of the lipid lowering potential further supporting their potential use in inflammatory based diseases. ${ }^{17,19-23}$ Similar to cardiovascular trials, in a randomized, double-blind, parallel group study in patients with COPD, Lee et al noted a reduction in CRP and IL-6 in patients treated with pravastatin compared to placebo, indicating the possibility for a role in modulating the inflammatory process of COPD. ${ }^{28}$ Initial trials of statins in COPD patients suggest potential benefit; however, many of the trials were retrospective in nature and did not address major outcome measures, such as hospitalization rates or mortality, indicating the need for further evaluation. ${ }^{24}$ The potential role of $\mathrm{HMG}-\mathrm{CoA}$ reductase inhibitors in COPD is reviewed in this article.

\section{Data selection}

A search of MEDLINE was performed using the search terms chronic obstructive pulmonary disease [drug therapy] and HMG-CoA reductase inhibitor [therapeutic use], from the time period January 2009 through May 2015, and limited to the English language and humans, to identify relevant literature. References from each identified article were also reviewed for applicable articles. Studies selected for inclusion in this review were randomized controlled, observational cohort, casecontrolled, and population-based analyses that included major outcome measures. Laboratory based studies were excluded.

\section{Retrospective studies and epidemiologic surveys}

Since 2009, several observational and epidemiological studies evaluating statin use in patients with COPD have been published and are summarized in Table 1. Bando et al conducted an epidemiological cross-sectional study in Japan between 2007 and 2008 which included 853 patients. Patients greater than 40 years of age who regularly visited one of the 16 participating primary health care facilities were included in this investigation. Participants were defined as either statin users or statin non-users. The outcome of interest in each cohort was airflow limitation defined as forced expiratory volume in 1 second/forced vital capacity $<70 \%$. Prevalence of airflow limitation was $2.25 \%$ in the statin use cohort compared to $10.5 \%$ in the statin non-use cohort $(P=0.01)$. Upon multivariate analysis to address potential confounding variables, the odds ratio for statin use as factor of airflow limitation was 0.28 (0.06-1.28). Ultimately the authors concluded that although airflow limitation was five times lower in the statin use cohort, the decrease in airflow limitation was not directly attributable to the potential disease modifying effects of statins. ${ }^{29}$

Lawes et al performed a population-based cohort study utilizing national data in New Zealand. A total of 1,687 patients aged 50-80 years admitted to a hospital with a COPD event were included. Groups were based on statin use prior to hospitalization and included 596 statin users and 1,091 non-statin users. The majority of statin users (91\%) were prescribed simvastatin, with atorvastatin prescribed in the remaining 9\%. As with other observational studies, accurate data on statin dose and duration of treatment were not available. Time to death from any cause over a 4-year follow-up from hospital discharge was the outcome of interest. Proportion of deaths was similar with 242 deaths $(40.6 \%)$ in the statin group and $429(39.3 \%)$ in the non-statin group. After adjusting for confounders using Cox's proportional hazards model, the hazard ratio (HR) of 0.69 (95\% confidence interval [CI] 0.58-0.84) favored statin use. ${ }^{30}$

Another observational study was conducted in Scotland by Sheng et al who used several linked databases to identify patients with a diagnosis of COPD and at least two total cholesterol measurements. Patients were classified into two cohorts, primary and secondary prevention of cardiovascular events. The primary prevention cohort included 1,274 patients, 617 in the statin group and 657 in the non-statin group. The secondary prevention cohort included 443 patients of which 292 were statin-treated and 151 were not. In the primary prevention cohort, association between statin use and cardiovascular events was not statistically significant. Statin use was associated with a $39 \%$ reduction in all-cause mortality 


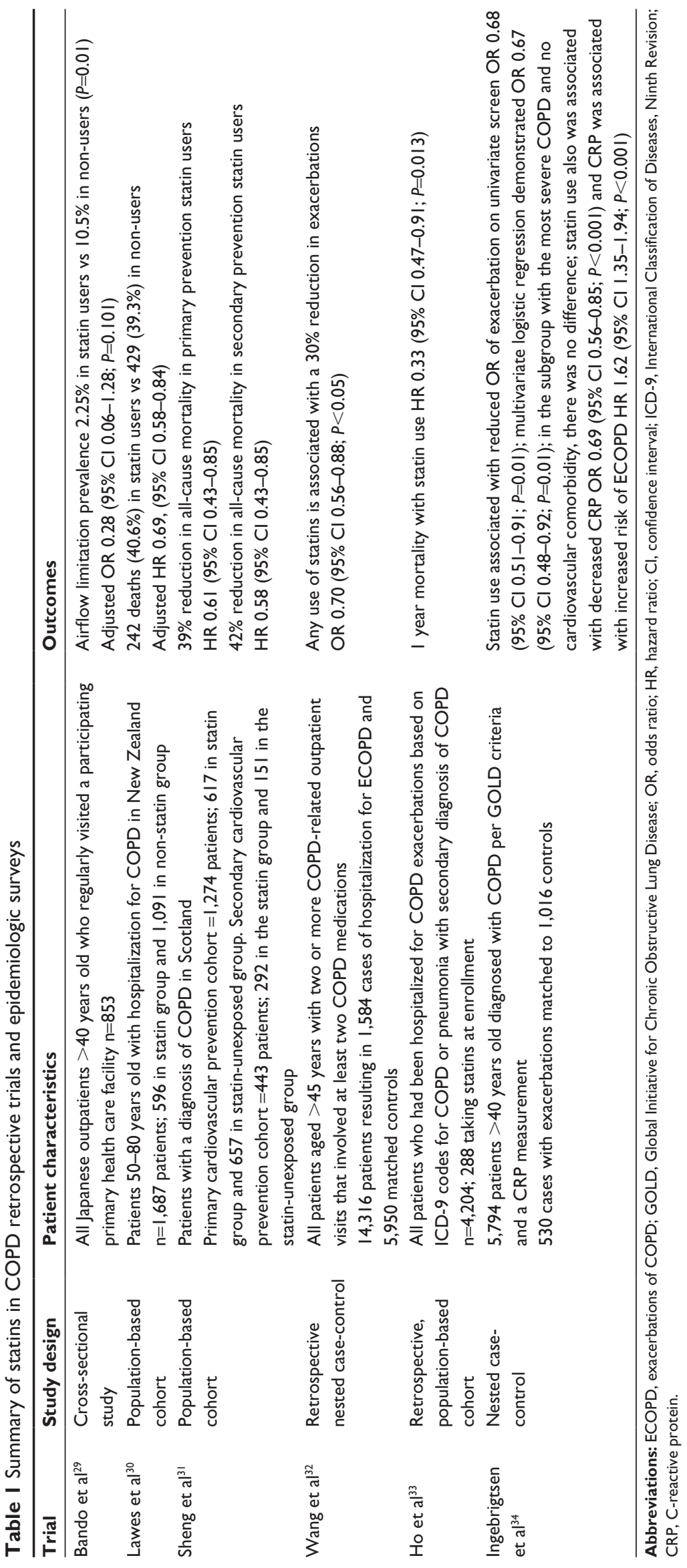


(HR $0.61,95 \%$ CI $0.43-0.85$ ). In the secondary prevention cohort, statin use was associated with a $65 \%$ reduction in cardiovascular events (HR 0.35, 95\% CI 0.15-0.87). Statin use also reduced risk for all-cause mortality by $42 \%$ (HR $0.58,95 \%$ CI $0.35-0.97)$. The authors concluded that statins improve all-cause mortality, but are not protective for primary prevention of cardiovascular events and death. ${ }^{31}$

A retrospective nested case-control study, using data from a Taiwan health insurance database, was conducted by Wang et al. Of 14,316 patients greater than 45 years old with COPD and two or more COPD-related outpatient visits and two or more COPD-related medications within 1 year, 1,584 cases with hospitalization for COPD exacerbation were matched to 5,950 controls. Statins, including atorvastatin, simvastatin, lovastatin, and fluvastatin, were prescribed in $9 \%$ of cases and $12.3 \%$ of controls. After multivariate logistic regression analysis, risk of COPD exacerbation requiring hospitalization was reduced $30 \%$ in patients with any use of statins (odds ratio [OR] 0.70, 95\% CI 0.56-0.88, $P<0.05$ ). When stratified by statin dose and duration, the risk was not decreased with increasing duration of statin use, but risk decreased in a dose-dependent manner. Smoking status was not measured, however sensitivity analyses suggest it was unlikely to confound the results. ${ }^{32}$

In one of the most recent investigations, published in 2014, Ho et al using a retrospective, population-based cohort, sought to determine if statin use was associated with decreased mortality during hospitalization for COPD exacerbation and at 1 year after hospitalization. Patients were selected from the Longitudinal Health Insurance Database of Taiwan from 2005 to 2007 and followed until the end of 2008. All patients hospitalized for COPD exacerbations based on International Classification of Diseases, Ninth Revision (ICD-9) codes for COPD, or ICD-9 codes for pneumonia with a secondary diagnosis of COPD, were included. Of the 4,204 patients studied, 288 patients were taking statin medications at the time of hospitalization. Statin use was not a predictor of decreased in-hospital mortality based on multivariate analysis; however, 322 patients were receiving statins by hospital discharge and statin use was associated with increased survival at 1 year (HR 0.33 [0.47-0.91]). Based on the results of this investigation, the authors concluded that statins may have a dual cardiopulmonary protective effect and likely improve outcomes for patients with severe COPD exacerbations. ${ }^{33}$

In the most recent investigation assessing statin use in COPD, Ingebrigtsen et al in a nested case control consisting of 5,794 individuals, matched 530 patients with COPD exacerbation to 1,016 controls. The outcome of interest, exacerbation of COPD, after multivariate logistic regression analysis, occurred less frequently in patients taking statins OR 0.67 (0.48-0.92). However, there was no benefit to statin use in the subgroup with the most severe COPD and no cardiovascular co-morbidity. Additionally, statin use was associated with a reduction in CRP, OR 0.69 (0.56-0.85) and CRP was associated with an increased risk of COPD exacerbation, HR 1.62 (1.35-1.94). Based on this data, the authors concluded that statin use was in fact associated with a reduced risk of COPD exacerbation; however, based on the subgroup analysis, this benefit is likely to be seen only in patients with COPD in the setting of co-morbid cardiovascular disease. ${ }^{34}$

\section{Prospective and randomized clinical trials}

One of the few prospective studies examining the role of statins in COPD was conducted by Bartziokas et al and published in 2011. All consecutive patients admitted to either of two tertiary hospitals with a diagnosis of COPD exacerbation, as defined by the Global Initiative for Chronic Obstructive Lung Disease (GOLD) guidelines, were eligible for inclusion. Patients were stratified based on statin use vs statin non-use and followed for 1 year. During this period, 30-day and 1-year mortality did not differ between groups. However, exacerbations of COPD (ECOPD) were less frequent in patients taking statins compared to those not taking statins $(2.1 \pm 2.7 \mathrm{vs}$ $2.8 \pm 3.2 \mathrm{ECOPD} /$ patient respectively; $P=0.037$ ). Additionally, the use of statins prolonged the time to next ECOPD (HR 0.656 [0.454-0.946]) and next severe ECOPD (HR 0.608 [0.381-0.972]), even after proper adjustments for significant confounders. Finally, the use of statins was related to a more profound improvement in health related quality of life at 2 , 6 , and 12 months after discharge $(P<0.001){ }^{35}$

In the largest population-based cohort to date, statin use was associated with a lower risk of COPD-related hospitalization compared to statin non-use. Huang et al matched 6,252 newly diagnosed COPD patients receiving statins for hyperlipidemia to 12,469 newly diagnosed COPD patients not taking statin medications. Diagnosis and study inclusion were based on ICD-9 codes corresponding with COPD of any disease severity. The outcome of interest was hospitalization with a principle diagnosis of COPD exacerbation, based on ICD-9 code. During an average follow-up of 4.58 years, 508 $(8.1 \%)$ statin-treated patients were hospitalized compared to $1,324(10.6 \%)$ patients from the control group. After adjusting for differences in baseline characteristics, statin use was 
associated with a decreased risk of hospitalization for COPDrelated complications (HR $0.66[0.60-0.74]) .{ }^{36}$

Lahousse et al performed a prospective nested case control analysis using data from the Rotterdam Study, a population-based cohort study conducted in the Netherlands. From a population of 7,983 individuals, 363 patients with COPD who died during follow-up were matched to 2,345 controls. Risk of all-cause mortality was stratified according to statin use and baseline serum level of CRP. After adjusting for confounders using conditional logistic regression, there was a $39 \%$ reduced risk of all-cause mortality when statins were used longer than 2 years (OR 0.61, 95\% CI 0.38-0.99, $P=0.045$ ). Further reduction was seen in the long-term statin group when baseline CRP was greater than $3 \mathrm{mg} / \mathrm{L}$ (OR $0.22,95 \% \mathrm{CI} 0.06-0.74, P=0.015$ ). This finding suggests that statin therapy may be most effective in those with evidence of increased systemic inflammation. ${ }^{37}$

STATCOPE was the largest, prospective clinical trial designed to elucidate if statin therapy reduced exacerbations in patients with moderate to severe COPD as defined by the GOLD guidelines. Eight hundred and fifty-five patients not requiring statin therapy for other indications were enrolled, 433 of whom received simvastatin $40 \mathrm{mg}$ daily and 452 of whom received matching placebo. The mean number of exacerbations per person-year was $1.36 \pm 1.61$ in the simvastatin group compared to $1.39 \pm 1.73$ in the placebo group $(P=0.54)$. Similarly, time to first exacerbation showed no statistical difference between the simvastatin and placebo groups, 223 (95\% CI, 195-275) days and 231 (95\% CI, 193-303) days respectively. Due to futility in terms of minimal to no differences in number of exacerbations per person-year and time to first exacerbation, as well as severity of exacerbation, number of acute cardiovascular events, quality of life and changes in spirometric variables, STATCOPE was stopped early after 43 months of follow-up. Ultimately the authors concluded that simvastatin had little treatment effect in reducing COPD exacerbations. Notably, the dose of simvastatin used in this trial may have been insufficient to produce significant anti-inflammatory effects and may explain the differences in treatment effect observed in STATCOPE compared to multiple retrospective investigations. ${ }^{38}$

Another randomized, controlled trial, RODEO, was conducted by Neukamm et al to determine whether treatment with rosuvastatin improved vascular function in patients with COPD in the absence of standard indications for statin therapy. Patients with diabetes, history of coronary artery disease, cerebrovascular and peripheral vascular disease were excluded. Ninety-nine patients were randomized, the majority of whom were considered low risk for cardiovascular disease; 49 received rosuvastatin $10 \mathrm{mg}$ daily and 50 received daily matching placebo for 12 weeks. No significant changes from baseline in vascular function or pulmonary function were observed. Secondary outcomes were changes from baseline of inflammatory markers CRP and IL-6, of which significant improvement was seen in the rosuvastatin group. The authors concluded that short-term treatment with rosuvastatin in patients with stable COPD did not improve vascular function or pulmonary function, but did reduce CRP levels and attenuate the rise of IL-6. As with the STATCOPE study, the statin dose used may have been too low to produce significant anti-inflammatory effects and the length of treatment was likely too short to produce a demonstrable effect on clinical outcomes..$^{39}$ The summary of the prospective and randomized clinical trials ${ }^{35-39}$ can found in Table 2.

\section{Conclusion}

Although there is a growing body of retrospective and prospective cohort evidence suggesting that statin use in COPD is associated with improvements in many aspects of the disease process, including reduction in mortality, hospitalizations for COPD, ECOPD, severity of exacerbations, inflammatory mediators, and improved health related quality of life, similar conclusions have yet to be borne out in prospective, randomized trials. The absence of confirmatory data from randomized, prospective clinical trials may stem from the use of statin doses insufficient to produce the pleiotropic effects necessary to modify the COPD disease process. Bonnet et al comparing atorvastatin $80 \mathrm{mg}$ to atorvastatin $10 \mathrm{mg}$, demonstrated a dose-dependent effect of atorvastatin on lowering CRP. ${ }^{19}$ With this data in mind, future investigations exploring the role of statins in COPD should consider the use of high dose statins, namely atorvastatin $80 \mathrm{mg}$ and rosuvastatin $40 \mathrm{mg}$.

Given the lack of confirmatory prospective data, the most recent iteration of the GOLD guidelines provide no guidance regarding the use of statins for COPD. Additionally, the 2015 joint guideline from American College of Chest Physicians and Canadian Thoracic Society for prevention of acute ECOPD recommends against the routine use of statins. ${ }^{40}$ Authors of the guideline do remark however, that many patients with COPD will require statins based on cardiovascular comorbidities, and should be treated accordingly. Due to the highest level of evidence not supporting the use of statins in COPD, and prominent guidelines recommending against routine use, it is the opinion of this panel that statins not be routinely prescribed for management of COPD; however, in patients with multiple COPD exacerbations 


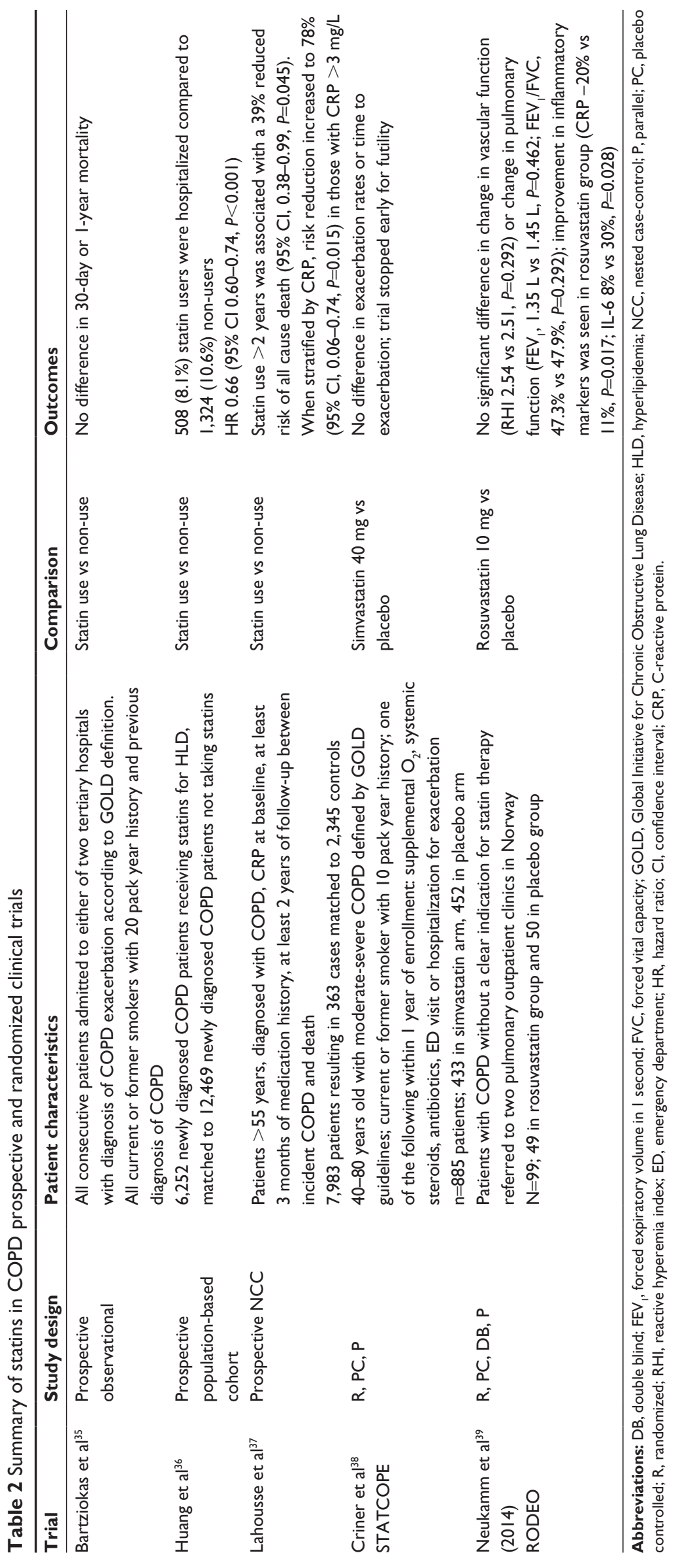


despite optimal medical management, especially for those with cardiovascular comorbidities, statin use may provide significant benefit. In order to optimize potential benefits of statin therapy in this patient population, high dose therapy with atorvastatin $80 \mathrm{mg}$ or rosuvastatin $40 \mathrm{mg}$ is warranted to take full advantage of the pleiotropic effects. Atorvastatin and rosuvastatin may be started at the respective doses without titration; however, caution is warranted when using high dose statins due to the potential increased risk for statin-related myalgias and myopathies.

\section{Disclosure}

The authors report no conflicts of interest in this work.

\section{References}

1. Global Initiative for Chronic Obstructive Lung Disease [homepage on the Internet]. Global strategy for the diagnosis, management and prevention of chronic obstructive pulmonary disease. Global Initiative for Chronic Obstructive Lung Disease; 2015 [updated 2015]. Available from: http://www.goldcopd.org/. Accessed August 27, 2015.

2. van Eeden SF, Hogg JC. The response of human bone marrow to chronic cigarette smoking. Eur Respir J. 2000;15(5):915-921.

3. Saetta M. Airway inflammation in chronic obstructive pulmonary disease. Am J Respir Crit Care Med. 1999;160(5 Pt 2):S17-S20.

4. Maestrelli P, Saetta M, Mapp CE, Fabbri LM. Remodeling in response to infection and injury airway inflammation and hypersecretion of mucus in smoking subjects with chronic obstructive pulmonary disease. $\mathrm{Am} \mathrm{J}$ Respir Crit Care Med. 2001;164(10 Pt 2):S76-S80.

5. Rennard SI. Inflammation and repair processes in chronic obstructive pulmonary disease. Am J Respir Crit Care Med. 1999;160(5 Pt 2):S12-S16.

6. Cosio MG, Guerassimov A. Chronic obstructive pulmonary disease inflammation of small airways and lung parenchyma. Am J Respir Crit Care Med. 1999;160(5 Pt 2):S21-S25.

7. Shapiro SD. The macrophage in chronic obstructive pulmonary disease. Am J Respir Crit Care Med. 1999;160(5 Pt 2):S29-S32.

8. Hill AT, Bayley D, Stockley RA. The interrelationship of sputum inflammatory markers in patients with chronic bronchitis. Am J Respir Crit Care Med. 1999;160(3):893-898.

9. Hogg JC. Pathophysiology of airflow limitation in chronic obstructive pulmonary disease. Lancet. 2004;364(9435):709-721.

10. Stockley RA. Neutrophils and the pathogenesis of COPD. Chest. 2002; 121(5 suppl):151S-155S.

11. Oudijk EJ, Lammers JW, Koenderman L. Systemic inflammation in chronic obstructive pulmonary disease. Eur Respir J Suppl. 2003;22(46):5s-13s.

12. Thorleifsson SJ, Margretardottir OB, Gudmundsson G, et al. Chronic airflow obstruction and markers of systemic inflammation: results from the BOLD study in Iceland. Respir Med. 2009;103(10):1548-1553.

13. Gan WQ, Man SF, Senthilselvan A, Sin DD. Association between chronic obstructive pulmonary disease and systemic inflammation: a systematic review and a meta-analysis. Thorax. 2004;59(7):574-580.

14. Karadag F, Kirdar S, Karul AB, Ceylan E. The value of C-reactive protein as a marker of systemic inflammation in stable chronic obstructive pulmonary disease. Eur J Intern Med. 2008;19(2):104-108.

15. Karadag F, Karul AB, Cildag O, Yilmaz M, Ozcan H. Biomarkers of systemic inflammation in stable and exacerbation phases of COPD. Lung. 2008;186(6):403-409.

16. Sin DD, Man SF. Why are patients with chronic obstructive pulmonary disease at increased risk of cardiovascular diseases? The potential role of systemic inflammation in chronic obstructive pulmonary disease. Circulation. 2003;107(11):1514-1519.

17. Ridker PM, Rifai N, Pfeffer MA, Sacks F, Braunwald E. Long-term effects of pravastatin on plasma concentration of C-reactive protein. Circulation. 1999;100(3):230-235.
18. Pruefer D, Makowski J, Schnell M, et al. Simvastatin inhibits inflammatory properties of Staphylococcus aureus $\alpha$-toxin. Circulation. 2002; 106(16):2104-2110.

19. Bonnet J, McPherson R, Tedgui A, et al. Comparative effects of 10-mg versus 80 -mg atorvastatin on high-sensitivity C-reactive protein in patients with stable coronary artery disease: results of the CAP (comparative atorvastatin pleiotropic effects) study. Clin Ther. 2008;30(12): 2298-2313.

20. Jialal I, Stein D, Balis D, Grundy SM, Adams-Huet B, Devaraj S. Effect of hydroxymethyl glutaryl coenzyme A reductase inhibitor therapy on high sensitive C-reactive protein levels. Circulation. 2001;103(15): 1933-1935.

21. Kinlay S, Schwartz GG, Olsson AG, et al. High-dose atorvastatin enhances the decline in inflammatory markers in patients with acute coronary syndromes in the MIRACL study. Circulation. 2003;108(13): $1560-1566$.

22. Albert MA, Danielson E, Rifai N, Ridker PM; PRINCE Investigators. Effect of statin therapy on C-reactive protein levels. The pravastatin inflammation/CRP evaluation (PRINCE): a randomized trial and cohort study. JAMA. 2001;286(1):64-70.

23. Balk EM, Lau J, Goudas LC, et al. Effects of statins on nonlipid serum markers associated with cardiovascular disease. Ann Intern Med. 2003;139(8):670-682.

24. Janda S, Park K, FitzGerald J, Etminan M, Swiston J. Statins in COPD: a systematic review. Chest. 2009;136(3):734-743.

25. Pruefer D, Scalia R, Lefer AM. Simvastatin inhibits leukocyteendothelial cell interactions and protects against inflammatory processes in normocholesterolemic rats. Arterioscler Thromb Vasc Biol. 1999; 19(12):2894-2900.

26. Morimoto K, Janssen WJ, Fessler MB, et al. Lovastatin enhances clearance of apoptotic cells (efferocytosis) with implications for chronic obstructive pulmonary disease. Immunol. 2006;176(12):7657-7665.

27. Hothersall E, McSharry C, Thomson NC. Potential therapeutic role for statins in respiratory disease. Thorax. 2006;61(8):729-734.

28. Lee TM, Lin MS, Chang NC. Usefulness of C-reactive protein and interleukin- 6 as predictors of outcomes in patients with chronic obstructive pulmonary disease receiving pravastatin. Am J Cardiol. 2008; 101(4):530-535.

29. Bando M, Miyazawa T, Shinohara H, Owada T, Terakado M, Sugiyama Y. An epidemiological study of the effects of statin use on airflow limitation in patients with chronic obstructive pulmonary disease. Respirology. 2012;17(3):493-498.

30. Lawes C, Thornley S, Young R, et al. Statin use in COPD patients is associated with a reduction in mortality: a national cohort study. Prim Care Respir J. 2012;21(1):35-40.

31. Sheng X, Murphy M, MacDonald T, et al. Effect of statins on total cholesterol concentrations, cardiovascular morbidity, and all-cause mortality in chronic obstructive pulmonary disease: a population-based cohort study. Clin Ther. 2012;34(2):374-384.

32. Wang M, Lo Y, Tsai C, et al. Statin use and risk of COPD exacerbation requiring hospitalization. Am J Med. 2013;126(7):598-606.

33. Ho TW, Tsai YJ, Ruan SY, et al. In-hospital and one-year mortality and their predictors in patients hospitalized for first-ever chronic obstructive pulmonary disease exacerbations: a nationwide population-based study. PLoS One. 2014;9(12):e114866.

34. Ingebrigtsen T, Marott J, Nordestgaard B, Lange P, Hallas J, Vestbo J. Statin use and exacerbations in individuals with chronic obstructive pulmonary disease. Thorax. 2015;70(1):33-40.

35. Bartziokas K, Papaioannou A, Minas M, et al. Statins and outcome after hospitalization for COPD exacerbation: a prospective study. Pulm Pharmacol Ther. 2011;24(5):625-631.

36. Huang CC, Chan WL, Chen YC, et al. Statin use and hospitalization in patients with chronic obstructive pulmonary disease: a nationwide population based cohort study in Taiwan. Clin Ther. 2011;33(10):1365-1370.

37. Lahousse L, Loth D, Joos G, et al. Statins, systemic inflammation and risk of death in COPD: The Rotterdam study. Pulm Pharmacol Ther. 2013;26(2):212-217. 
38. Criner G, Connett J, Aaron SD, et al. Simvastatin for the prevention of exacerbations in moderate to severe COPD. N Engl J Med. 2014; 370(23):2201-2210.

39. Neukamm A, Hoiseth A, Einvik G, et al. Rosuvastatin treatment in stable chronic obstructive pulmonary disease (RODEO): a randomized controlled trial. J Intern Med. 2015;278(1):59-67.
40. Criner G, Bourbeau J, Diekemper R, et al. Prevention of acute exacerbations of COPD - American College of Chest Physicians and Canadian Thoracic Society Guideline. Chest. 2015;147(4):894-942.

\section{Publish your work in this journal}

The International Journal of COPD is an international, peer-reviewed journal of therapeutics and pharmacology focusing on concise rapid reporting of clinical studies and reviews in COPD. Special focus is given to the pathophysiological processes underlying the disease, intervention programs, patient focused education, and self management protocols.

\section{Dovepress}

This journal is indexed on PubMed Central, MedLine and CAS. The manuscript management system is completely online and includes a very quick and fair peer-review system, which is all easy to use. Visit $\mathrm{http} / / / \mathrm{www}$.dovepress.com/testimonials.php to read real quotes from published authors. 